\title{
La electricidad como factor de competitividad: Evidencia empírica en el mercado de clientes libres en el contexto del gas de Camisea
}

Miguel Peralta Neyra ${ }^{1}$

Recibido: 26/09/2019 Aceptado: 18/04/2021 Publicado: 26/07/2021

\begin{abstract}
RESUMEN
La explotación del gas de Camisea supuso beneficios para las industrias con respecto a las tarifas eléctricas de las que son ingentes consumidoras. El mercado eléctrico nacional se rige por tres precios: precio en barra, costo marginal y precio de cliente libre. En este estudio se pretende establecer si la ventaja de un menor precio del gas natural se refleja en el precio de los clientes libres, que son aquellos que pueden negociar directamente su tarifa eléctrica con los generadores o distribuidores de electricidad. Esto se logró mediante un análisis de series de tiempo con modelos econométricos en el que se vincularon los tres precios del mercado eléctrico nacional y que indicó la correlación existente entre cada uno de ellos. Se efectuó la revisión de los modelos propuestos, su análisis correlacional y el análisis de autocorrelación de los residuos, lo que dio como resultado una correlación fuerte entre el precio del mercado libre y su primer rezago, hecho que desvincula una correlación con los otros precios; por tanto, no hay un traslado real del precio del gas en beneficio de una mejor tarifa para el cliente libre.
\end{abstract}

Palabras clave: matriz energética; consumidores no regulados; precio de barra; costo.

\section{INTRODUCCIÓN}

Con la promulgación de la Ley de Concesiones Eléctricas (Ley $\mathrm{N}^{\circ} 25844,19$ de noviembre de 1992) se cambió el rol del Estado en el sector eléctrico, ya que pasó de una función de administración a una de supervisión. Con este fin, se reformularon y fortalecieron las funciones de entes reguladores ya existentes y posteriormente se crearon otros con tareas específicas. En la ley se especifican aspectos tales como la nueva estructura del sector, las actividades y mercados existentes, y los regímenes de precios. Asimismo, se definieron las funciones y la estructura de la Comisión de Tarifas Eléctricas (hoy llamada Gerencia Adjunta de Regulación Tarifaria, GART, perteneciente a Osinergmin), los procedimientos a seguirse para las concesiones, los derechos y obligaciones de los concesionarios, y las funciones del organismo encargado de la operación eficiente del sistema (COES). Paralelamente, se definieron las transacciones sujetas a regulación de precio, así como los procedimientos para la fijación de las tarifas.

Si bien con la Ley de Concesiones Eléctricas se desintegró el monopolio estatal, la privatización posterior debía asegurar que no se dieran las figuras de una integración vertical y concentración horizontal; así, la ley fue diseñada con la asignación de esta responsabilidad al organismo regulador Osinergmin. Por otro lado, algunos autores ven la integración vertical como una ventaja por la posibilidad que ofrece de compartir sinergias y riesgos (Viscusi, Vernon y Harrington, 2005). La menor competencia, ligada a las menores opciones de elección de los consumidores y al aprovechamiento monopólico de una serie de especificidades como la localización, inversiones idiosincráticas, etc., no solo afecta al consumidor, sino que distorsiona el mercado. Con relación a la concentración horizontal, a partir del año 2012 el índice de Herfindahl-Hirschman (HHI) fue menor a 1800, lo que significa que este mercado era moderadamente concentrado; cabe precisar que la tendencia del HHI es a la disminución (Cuadros y López, 2016).

Graduado en Ingeniería Mecánica por la Pontificia Universidad Católica del Perú (Lima, Perú). Especialista en motores de gas natural y proyectos de generación eléctrica. Actualmente, es docente en tópicos de energía de la Pontificia Universidad Católica del Perú y asesor independiente en proyectos de gas natural (Lima, Perú).

ORCID: https://orcid.org/0000-0002-7769-7606

Autor de correspondencia: peralta.m@pucp.pe 
El precio de la energía eléctrica que obtienen los clientes libres, también denominado precio no regulado (Morandé y Soto, 1996), se establece a través de los contratos que adquieren en la negociación con los oferentes, que en este caso son los generadores y distribuidores del mercado eléctrico (Dammert, Mollinelli y Carbajal, 2011). La mayoría de estos clientes libres son grandes compañías del sector minero, fundiciones, cementeras, químicas, metalúrgicas, industriales, etc. El precio que pagan estos clientes reviste de una gran importancia, ya que es justamente en este segmento de clientes donde la competencia tiene, de acuerdo con la propia regulación, un mayor potencial.

Por su parte, los clientes regulados, aquellos cuya tarifa es regulada, se encuentran bajo el amparo de Osinergmin, quien fija las tarifas máximas de generación, transmisión y distribución. Los precios del mercado regulado están relacionados con los precios en barra, precio que los generadores o distribuidores aseguran hasta una barra física o punto de suministro localizado dentro de su red, y con los costos marginales. Ambos precios, los de barra (Osinergmin, 2017) y costo marginal (Resolución de Consejo Directivo Osinergmin N ${ }^{\circ}$ 013-2016OS/CD, 2016), están influenciados por el valor del combustible, ya que genera la diferenciación de la inversión de tecnologías y costos; por ejemplo, las hidroeléctricas requieren de una gran inversión inicial con un bajo valor del "combustible" (el agua represada) para su operación, mientras que los turbogeneradores a diésel son de baja inversión de capital con un costo de combustible (hidrocarburo) mayor frente al recurso hídrico.

Osinergmin ha establecido que la tarifa de los clientes regulados no debe diferir ni en más ni en menos del $10 \%$ del promedio ponderado de los precios de las licitaciones en las que participan los clientes libres, es decir, los precios del mercado regulado son sensibles al precio del mercado libre (Resolución del Consejo Directivo Osinergmin N ${ }^{\circ} 273-2010-O S /$ $C D, 2010)$. De esta manera, es razonable pensar que, si los precios obtenidos por los clientes libres sufrieran una manipulación, este efecto se trasladaría al precio regulado (De La Cruz y García, 2002). Tomemos debida nota de que los mismos oferentes de energía eléctrica del mercado de clientes libres pueden, y de hecho son, suministradores de energía del mercado regulado. Se investiga cómo se están comportando los precios del mercado libre respecto a los precios que forman el mercado regulado (precio regulado) y si el gas de Camisea realmente contribuye a que el sector de clientes libres sea más competitivo.
Es común escuchar que el gas de Camisea, desde sus inicios, se considera una fuente de energía que mejora y mejorará la economía del país; sin embargo, no ha habido un estudio serio sobre su influencia en la mejora de los costos de producción en las industrias, más aún, sabiendo que la energía eléctrica es un insumo con gran peso específico en el costo de producción.

Dado que la energía eléctrica constituye un insumo importante dentro del proceso productivo de la mayoría de las industrias, principalmente en la minería, fundición, refinería, etc., se quiere establecer si el ingreso del gas natural en la nueva matriz energética peruana realmente contribuye a que la industria en general se esté beneficiando de ello y esté logrando ser más competitiva mediante tarifas menores por el hecho de que el gas natural es un combustible más barato que los demás hidrocarburos. Se vuelve a enfatizar que los precios que se establecen en el mercado eléctrico son: los precios de clientes libres, los precios regulados (que están en función de los precios en barra y el costo marginal) y el costo marginal. Como el precio en barra y el costo marginal están fuertemente influenciados por el costo del combustible, surge la necesidad de saber si los beneficios de un menor precio del gas están siendo trasladados a los clientes libres.

El objetivo de este trabajo es investigar si el gas natural, como nueva fuente en la matriz energética, contribuye a mejorar la competitividad de la industria peruana. Para este propósito se van a establecer las relaciones significativas de los precios del mercado eléctrico que influyen en el precio del mercado de clientes libres. También se busca determinar en qué nivel influyen los precios del cliente libre rezagados en el precio del cliente libre, y en qué nivel el costo marginal presente y sus rezagos influyen en el precio del cliente libre.

Este trabajo se justifica en la investigación de las relaciones que se suceden con los precios del mercado eléctrico. Se busca establecer si hay un traslado del beneficio de contar con un hidrocarburo más barato, como el gas natural, hacia una industria que es intensiva en el uso de la energía eléctrica en su proceso productivo. También se mostrará qué influencia tienen los precios en barra, el costo marginal y sus rezagos, incluyendo el propio precio del cliente libre, en el precio del cliente libre. Esta investigación se puede extender a otros mercados regulados como el de las telecomunicaciones, el servicio de agua potable y el transporte. 


\section{METODOLOGÍA}

En vista de que esta investigación tiene como elemento de análisis la influencia del desarrollo del gas de Camisea en el mercado de clientes libres, se debe especificar cuándo se empezó a contar con este hidrocarburo para la generación termoeléctrica. El proyecto del gas de Camisea concluyó con la puesta en marcha de la estación de compresión de Malvinas y las estaciones de bombeo de LNG en agosto del 2004 (El Comercio, 2014). Por su parte, las generadoras que a la fecha del presente estudio emplean gas de Camisea empezaron a operar a finales del 2004, siendo una de las más importantes la Planta de Etevensa en Ventanilla, que inició sus operaciones con ciclo simple y luego se consolidó en el segundo semestre del año 2006 con el ingreso de su ciclo combinado. Adicionalmente los grandes termogeneradores localizados en Chilca, que son alimentados exclusivamente por el gas proveniente de Camisea entraron en operación comercial como Kallpa I y Chilca I en el 2006 y 2007 con sus turbinas a gas T1 y T2 respectivamente. Lo anteriormente expuesto permite tener la confianza de que la muestra a elegir como población de estudio es la información referente del mercado eléctrico a partir del año 2008 en adelante, donde ya se consolida y estabiliza la operación, despacho y el mercado mismo.

Esta investigación está basada en el análisis de documentos proporcionados por Osinergmin y el COES, que son las autoridades competentes que monitorean y preparan las estadísticas del desempeño del mercado eléctrico en el Perú.

La información se recogió de la siguiente manera:

Precio del mercado libre. Se accedió a la página web http://srvgart07.osinerg.gob.pe/SICLI/principal. aspx, luego a la opción "Evolución del Mercado Libre", se desplegó el menú de "Variable" y se seleccionó "Precio medio (ctm/S/./kW.h)"; seguidamente se desplegó el menú "Clasificado por" y se seleccionó "Mercado Libre"; para finalizar, se seleccionó el periodo de consulta.

Precio en barra y costo marginal. Se accedió a la página web http://www.coes.org.pe/Portal/Publicaciones/Estadisticas/, luego, a "Publicaciones" y a "Estadísticas Anuales"; se ingresó a la carpeta signada con el año 2017, después a la carpeta 01_EXCEL, a la subcarpeta 16_COSTOS MARGINALES DE CORTO PLAZO, y finalmente se ingresó al archivo Gráfico No 16.3_ COSTO MARGINAL PONDERADO Y TARIFA EN BARRA MENSUAL.
Esta investigación es de tipo cuantitativa, inductiva, básica, longitudinal, correlacional y no experimental. Las unidades de análisis se toman de los reportes de valores del mercado eléctrico peruano a través del Portal del Sistema de Usuarios Libres de Osinergmin (http://srvgart07.osinerg.gob.pe/SICLI/ principal.aspx) y del portal del COES (http://www. coes.org.pe/Portal/Publicaciones/Informes/).

Se presentan a continuación tres modelos correlacionales que se aplica a este estudio y que vinculan los precios que se dan en el mercado eléctrico peruano:

- Modelo 1 In $\mathrm{PL}_{\mathrm{t}}=\alpha+\beta \ln \mathrm{PB}_{\mathrm{t}}+\varepsilon_{\mathrm{t}}$

- Modelo 2 In PLt $=\alpha+\beta^{*} \ln \mathrm{CMgt}+\varepsilon t$

- Modelo $3 \quad \operatorname{InPL} L_{t}=\alpha+\beta^{*} \ln P L_{t-1}+\varepsilon_{t}$

Donde:

- $\alpha, \beta$ son coeficientes.

- $\varepsilon_{\mathrm{t}} \quad$ está definido como un shock aleatorio o estocástico.

- In es la función logaritmo natural.

- $\mathrm{PL}_{t}$ es el precio del mercado libre en el periodo t.

- $\mathrm{PL}_{\mathrm{t}-\mathrm{n}}$ es el precio del mercado libre con rezago $\mathrm{n}$.

- $\mathrm{PB}_{t}$ es el precio de barra en el periodo $t$.

- $\mathrm{CMg}_{\mathrm{t}}$ es el logaritmo natural del costo marginal en el periodo $t$.

Si la variabilidad de los datos crece con el nivel de los mismos, es decir, si hay cambios en los datos por los que la serie de tiempo fluctúa verticalmente de un periodo a otro, (por ejemplo, si de un año a otro el mínimo y máximo de la serie incrementa o disminuye), será necesario hacer una corrección, ya que representaría un problema; esto suele hacerse mediante una transformación logarítmica, aplicando logaritmos naturales a las observaciones y consiguiendo la estacionariedad en la varianza (Chong y Aguilar, 2016).

La población de estudio es el mercado eléctrico peruano, y para el tamaño de la muestra se han seleccionado los precios y costos del mercado eléctrico desde febrero del 2008 hasta diciembre del 2016. Estos valores se encuentran en la Tabla 1.

\section{RESULTADOS}

Para el análisis correlacional se ha empleado el software IBM SPSS Statistics 23 y se han considerado las pruebas de correlación de Pearson y $\mathrm{R}^{2}$ más la 
Tabla 1. Precios del mercado libre, precio en barra y costo marginal (ctv $\$ / k W . h$ ) del periodo febrero de 2008 a diciembre de 2016.

\begin{tabular}{|c|c|c|c|c|c|c|c|}
\hline Mes/año & Mercado Libre & $\begin{array}{c}\text { Regulado barra } \\
\text { Lima }\end{array}$ & $\begin{array}{c}\text { Costo } \\
\text { marginal }\end{array}$ & Mes/año & Mercado libre & $\begin{array}{c}\text { Regulado barra } \\
\text { Lima }\end{array}$ & $\begin{array}{c}\text { Costo } \\
\text { marginal }\end{array}$ \\
\hline Feb-08 & 49.4999 & 43.5792 & 18.3336 & Ago-12 & 58.5272 & 50.8816 & 35.0912 \\
\hline Mar-08 & 48.2512 & 45.0487 & 20.8422 & Sep-12 & 58.9011 & 52.1772 & 36.4090 \\
\hline Abr-08 & 50.2247 & 44.4016 & 20.9027 & Oct-12 & 59.5905 & 52.4412 & 28.7611 \\
\hline May-08 & 53.0576 & 44.1790 & 47.8629 & Nov-12 & 58.0696 & 51.3352 & 14.3501 \\
\hline Jun-08 & 61.7507 & 44.8090 & 148.8524 & Dic-12 & 57.7426 & 51.7424 & 13.7504 \\
\hline Jul-08 & 57.8397 & 40.1050 & 235.3823 & Ene-13 & 59.1261 & 52.2300 & 19.3484 \\
\hline Ago-08 & 68.9169 & 41.5437 & 157.8758 & Feb-13 & 60.2324 & 52.7082 & 31.4054 \\
\hline Sep-08 & 68.0654 & 39.8481 & 185.2081 & Mar-13 & 57.7833 & 51.3073 & 19.7176 \\
\hline Oct-08 & 54.5291 & 40.9945 & 63.3530 & Abr-13 & 59.7752 & 51.8077 & 18.7835 \\
\hline Nov-08 & 54.5909 & 41.1696 & 60.6917 & May-13 & 59.3034 & 50.2321 & 27.1427 \\
\hline Dic-08 & 55.0423 & 39.1783 & 81.7817 & Jun-13 & 58.1775 & 50.5006 & 26.6098 \\
\hline Ene-09 & 48.1669 & 37.7910 & 28.8897 & Jul-13 & 58.7349 & 50.2361 & 44.8607 \\
\hline Feb-09 & 49.6494 & 37.5074 & 42.3859 & Ago-13 & 58.5656 & 49.8932 & 34.7275 \\
\hline Mar-09 & 47.3418 & 37.8923 & 26.4559 & Sep-13 & 58.6253 & 48.2247 & 28.2676 \\
\hline Abr-09 & 45.7491 & 41.1450 & 25.4261 & Oct-13 & 58.0340 & 50.4502 & 19.4460 \\
\hline May-09 & 46.6875 & 39.7411 & 28.6713 & Nov-13 & 58.7762 & 50.2010 & 23.0011 \\
\hline Jun-09 & 49.5528 & 39.9566 & 65.7041 & Dic-13 & 58.6221 & 50.4372 & 24.8975 \\
\hline Jul-09 & 48.1972 & 39.8324 & 41.2193 & Ene-14 & 59.3035 & 50.1908 & 21.3909 \\
\hline Ago-09 & 47.2772 & 39.4824 & 33.8807 & Feb-14 & 60.1234 & 55.0746 & 29.8533 \\
\hline Sep-09 & 47.7056 & 40.1442 & 36.2248 & Mar-14 & 59.6457 & 54.4080 & 34.3143 \\
\hline Oct-09 & 46.8315 & 40.5292 & 19.7863 & Abr-14 & 60.7635 & 55.0021 & 28.1039 \\
\hline Nov-09 & 49.6828 & 40.7031 & 20.3717 & May-14 & 59.7786 & 54.8269 & 25.4199 \\
\hline Dic-09 & 53.2740 & 38.5393 & 17.2423 & Jun-14 & 60.6186 & 54.8215 & 30.9638 \\
\hline Ene-10 & 53.2165 & 39.0021 & 23.1519 & Jul-14 & 60.1494 & 54.6584 & 24.9118 \\
\hline Feb-10 & 53.7494 & 39.8040 & 24.5490 & Ago-14 & 61.0708 & 54.2496 & 27.4186 \\
\hline Mar-10 & 50.7876 & 39.1649 & 21.9672 & Sep-14 & 61.2980 & 53.7230 & 23.8568 \\
\hline Abr-10 & 53.8770 & 39.8972 & 16.6041 & Oct-14 & 60.9968 & 52.4992 & 17.9716 \\
\hline May-10 & 47.0575 & 37.0416 & 18.1607 & Nov-14 & 60.2288 & 50.0767 & 23.4544 \\
\hline Jun-10 & 47.9560 & 36.9624 & 20.4322 & Dic-14 & 59.7798 & 49.3496 & 15.1614 \\
\hline Jul-10 & 48.3525 & 37.1588 & 19.8814 & Ene-15 & 59.9644 & 48.8229 & 14.1122 \\
\hline Ago-10 & 48.8606 & 37.5823 & 22.8932 & Feb-15 & 60.9203 & 50.9184 & 16.2237 \\
\hline Sep-10 & 50.3119 & 38.0923 & 23.8426 & Mar-15 & 58.9541 & 49.6404 & 17.0768 \\
\hline Oct-10 & 49.3977 & 37.6483 & 24.2287 & Abr-15 & 59.5711 & 49.6373 & 13.1058 \\
\hline Nov-10 & 50.0032 & 37.6004 & 23.1013 & May-15 & 58.8955 & 55.9126 & 14.8277 \\
\hline Dic-10 & 50.0807 & 37.2586 & 18.7580 & Jun-15 & 59.0806 & 55.9179 & 16.9142 \\
\hline Ene-11 & 51.6746 & 37.7511 & 17.4892 & Jul-15 & 58.2973 & 58.0145 & 10.9362 \\
\hline Feb-11 & 54.5046 & 38.4420 & 21.7420 & Ago-15 & 57.9421 & 57.1395 & 21.4953 \\
\hline Mar-11 & 53.4987 & 37.6326 & 21.6263 & Sep-15 & 59.0000 & 58.0990 & 14.4870 \\
\hline Abr-11 & 55.0812 & 39.2423 & 17.9153 & Oct-15 & 58.0086 & 57.3931 & 14.2471 \\
\hline May-11 & 53.2369 & 41.8831 & 18.7874 & Nov-15 & 57.5160 & 55.5090 & 11.5861 \\
\hline Jun-11 & 53.2595 & 42.2603 & 25.8559 & Dic-15 & 56.0025 & 54.0816 & 11.3972 \\
\hline Jul-11 & 53.8045 & 42.4235 & 20.4480 & Ene-16 & 54.7017 & 56.6792 & 10.9870 \\
\hline Ago-11 & 54.0971 & 42.4162 & 31.5137 & Feb-16 & 53.6933 & 54.9479 & 12.4248 \\
\hline Sep-11 & 55.4634 & 42.7455 & 33.6255 & Mar-16 & 50.8376 & 55.8568 & 12.3632 \\
\hline Oct-11 & 53.5310 & 42.4366 & 27.0617 & Abr-16 & 52.0433 & 54.5576 & 13.2597 \\
\hline Nov-11 & 54.9701 & 43.2516 & 28.5765 & May-16 & Sin datos & Sin datos & 19.9034 \\
\hline Dic-11 & 53.7427 & 43.1351 & 21.5721 & Jun-16 & Sin datos & Sin datos & 60.3911 \\
\hline Ene-12 & 55.3760 & 43.4912 & 20.9235 & Jul-16 & 53.7055 & 55.1906 & 34.1345 \\
\hline Feb-12 & 56.1628 & 44.3116 & 23.7337 & Ago-16 & 53.3021 & 56.7516 & 18.9263 \\
\hline Mar-12 & 56.6856 & 43.6561 & 39.8348 & Sep-16 & 52.8905 & 56.3791 & 27.5588 \\
\hline Abr-12 & 56.1615 & 44.1915 & 26.6761 & Oct-16 & 51.5354 & 56.4675 & 17.9264 \\
\hline May-12 & 58.3578 & 49.7053 & 27.1751 & Nov-16 & 51.8879 & 58.8807 & 27.5979 \\
\hline Jun-12 & 59.0050 & 50.1692 & 45.5229 & Dic-16 & 50.9509 & 58.8439 & 23.0784 \\
\hline Jul-12 & 58.6341 & 51.0439 & 58.0489 & & & & \\
\hline
\end{tabular}

Fuente: Elaboración propia tomando como base la información de http://srvgart07.osinerg.gob.pe/SICLI/principal.aspx y http://www.coes. org.pe/Portal/Publicaciones/Estadisticas/ 
de Durbin Watson (Daza, 2016). Los resultados de cada uno de los modelos propuestos se encuentran en el Anexo A.

La Tabla 2 muestra los valores de los coeficientes, constantes y estadísticos que se consideraron para la presente evaluación.

Tabla 2. Resultado de los modelos propuestos en el software IBM SPSS Statistics 23.

\begin{tabular}{|l|c|c|c|}
\hline \multicolumn{1}{|c|}{ Valores } & \multicolumn{3}{c|}{ Modelos } \\
\hline$\alpha$ & 2.784 & 4.097 & 0.602 \\
\hline$\beta$ & 0.319 & -0.078 & 0.850 \\
\hline$R^{2}$ & 0.564 & 0.081 & 0.725 \\
\hline$R^{2}$ ajustado & 0.318 & 0.072 & 0.722 \\
\hline Pearson & 0.311 & -0.285 & 0.852 \\
\hline$F$ & 48.863 & 9.270 & 269.120 \\
\hline Durbin Watson & 0.387 & 0.093 & 2.133 \\
\hline
\end{tabular}

Fuente: Elaboración propia a partir de los resultados del software SPSS.

Una medida de la dependencia lineal entre dos variables aleatorias cuantitativas es explicada por el coeficiente de correlación de Pearson: cuando sus valores están más cercanos a 1 se evidencia una marcada relación entre las variables. De los tres modelos estudiados, se observó que el que mayor correlación tiene con las variables consideradas para su caso es el modelo tres, ya que fue el que obtuvo el mayor coeficiente de correlación de Pearson, y este resultado fue refrendado por el coeficiente de determinación $\mathrm{R}^{2}$. Este modelo, confrontado con los valores reales, produce residuos que también deben ser analizados para descartar una autocorrelación de ellos, así, si se encuentra autocorrelación entre los residuos, se puede asegurar que el modelo no es el adecuado para explicar las relaciones de las variables y se presume la ausencia de una variable no tomada en cuenta y que debe ser incluida. El estadístico Durbin Watson es el que muestra con certeza si los residuos de las muestras no están correlacionados y, para el modelo 3, este valor se encuentra en la zona de no autocorrelación por lo que el modelo presentado produce residuos no autocorrelacionados y explica mejor la relación de las variables en estudio. Como prueba de contraste para validar el modelo, se ejecutó el modelo ARIMA.

\section{DISCUSIÓN}

El modelo propuesto también debe mostrar que no hay autocorrelación, y para ello empleamos el estadístico Durbin Watson (DB) proporcionado por el mismo software SPSS (Gujarati, 1997). Según la Tabla 2, el DB de nuestro modelo es 2.132 y debe compararse con la hipótesis nula que indica que no hay autocorrelación para los valores de DB entre $d_{u}$ y $4-d_{u}$, que, para un $5 \%$ de significancia, da un valor de $d_{u}$ igual a 1.694 y un valor de $4-d_{u}$ igual a 2.346; por lo tanto, la hipótesis nula de no autocorrelación queda validada. EI modelo ARIMA proporciona un valor del estadístico Ljung Box de 0.529 , superior a la mínima aceptada de 0.05 , por lo que el modelo es válido.

\section{CONCLUSIONES}

Existe una desvinculación del precio del cliente libre con los precios en barra y los costos marginales, ya que, a pesar de que estos valores son dependientes del costo del combustible, el precio del cliente libre no está conectado con el precio del gas natural. El modelo que mejor explica la relación del precio del cliente libre con los precios del mercado eléctrico es, precisamente, el modelo 3 , que vincula el precio del cliente libre rezagado un periodo; en el estudio, ese periodo es de un mes.

Las grandes industrias deberían poder gozar de los mismos beneficios que los generadores que, por ejemplo, son capaces de generar su propia energía. Las tarifas de gas natural para consumo industrial y para generación se encuentran diferenciadas y, de la misma manera, los mayores consumidores de gas para generación tienen mejores tarifas. La desconcentración de las líneas de alta tensión, gracias a la autogeneración, puede mejorar la calidad del servicio mismo que es bastante pobre en zonas extremas de las líneas y, a su vez, puede reforzarse con la inyección de los excedentes de las industrias.

\section{REFERENCIAS BIBLIOGRÁFICAS}

[1] Chong, M., y Aguilar, R. (2016). Proyección de series de tiempo para el consumo de la energía eléctrica a clientes residenciales en Ecuador. Revista Tecnológica ESPOL, 29(1), 56-76.

[2] El Comercio (30 de abril de 2014). El recorrido de Camisea, un proyecto que esperó dos décadas. El Comercio.

[3] Cuadros, A., y López, G. (2015). Análisis de condiciones de competencia en el mercado de generación eléctrica en el Perú, periodo 2010 2014. Recuperado de https://www.coes.org.pe/ Portal/Publicaciones/Articulos/

[4] Dammert, A., Mollinelli, F. y Carbajal, M. A. (2011). Fundamentos Técnicos y Económicos 
del Sector Eléctrico Peruano. Lima, Perú: Osinergmin.

[5] Daza, J. (2016). Análisis Estadístico SPSS Statistics. Lima, Perú: Megabyte SAC.

[6] De La Cruz, R. y García, R. (Febrero de 2002). Mecanismos de Competencia en Generación de Energía y su impacto en la Eficiencia: El caso peruano.. Recuperado de https://cies. org.pe/sites/default/files/investigaciones/ mecanismos-de-competencia-en-generacionde-energia-y-su-impacto-en-la-eficiencia.pdf

[7] Gujarati, D. (1997). Econometría. Sante Fé de Bogotá, Colombia: McGraw-Hill.

[8] Ley $\mathrm{N}^{\circ} 25844$ (19 de noviembre de 1992). Ley de Concesiones Eléctricas. Diario Oficial El Peruano. Recuperado de https://www. osinergmin.gob.pe/cartas/documentos/ electricidad/normativa/LEY_CONCESIONES_ ELECTRICAS.pdf

[9] Morandé, F., y Soto, R. (1996). El Mercado de clientes no regulados en la industria eléctrica. ILADES/Georgetow .
[10] Osinergmin (16 de Marzo de 2017). Procedimiento de Fijación de Precios en Barra Periodo mayo 2017 - abril 2018.

[11] Resolución del Consejo Directivo Organismo Supervisor de la Inversión en Energía y Minería Osinergmin $N^{\circ}$ 273-2010-OS/CD. Aprueban "Procedimiento para la comparación de precios regulados". Diario Oficial El Peruano (2010). Recuperado de http://www.osinergmin.gob. pe/newweb/uploads/Publico/Resoluciones/ ConsejoDirectivo/2010/OSINERGMIN\%20 No.273-2010-OS-CD.pdf

[12] Resolución de Consejo Directivo Organismo Supervisor de la Inversión en Energía y Minería Osinergmin $\mathrm{N}^{\circ}$ 013-2016-OS/CD. Diario Oficial El Peruano. Recuperado de http://www. osinergmin.gob.pe/Resoluciones/pdf/2016/ OSINERGMIN\%20No.013-2016-OS-CD.pdf

[13] Viscusi, W., Harrington, Jr., J., y Vernon, J. (2005). Economics of Regulation and Antitrust. Cambridge, MA, Estados Unidos: The MIT Press. 
ANEXO

Anexo A. Resultados del software IBM SPSS Statistics 2 para los modelos propuestos.

Resumen de los modelos ${ }^{(\mathrm{e})}$

\begin{tabular}{|c|c|c|c|c|c|}
\hline Modelo & $\mathbf{R}$ & $\mathbf{R}$ cuadrado & $\begin{array}{c}\text { R cuadrado } \\
\text { ajustado }\end{array}$ & Pearson & Durbin-Watson \\
\hline 1 & $0.564^{(\mathrm{a})}$ & 0.318 & 0.311 & 0.564 & 0.387 \\
\hline 2 & $0.091^{(\mathrm{b})}$ & 0.008 & -0.001 & -0.091 & 2,232 \\
\hline 3 & $0.285^{\odot}$ & 0.081 & 0.072 & -0.285 & 0.093 \\
\hline 4 & $0.852^{(\mathrm{d})}$ & 0.725 & 0.722 & 0.852 & 2,133 \\
\hline
\end{tabular}

a. Predictores: (Constante), InPB

b. Predictores: (Constante), var(InPBt-1)

c. Predictores: (Constante), $\ln (\mathrm{CMg})$

d. Predictores: (Constante), In(PLt-1)

Coeficientes $^{(a)}$

\begin{tabular}{|c|c|c|c|c|c|c|}
\hline \multirow{2}{*}{\multicolumn{2}{|c|}{ Modelo }} & \multicolumn{2}{|c|}{ Coeficientes no estandarizados } & \multirow{2}{*}{$\begin{array}{c}\begin{array}{c}\text { Coeficientes } \\
\text { estandarizados }\end{array} \\
\text { Beta }\end{array}$} & \multirow{2}{*}{$\mathbf{T}$} & \multirow{2}{*}{ Sig. } \\
\hline & & B & Error estándar & & & \\
\hline \multirow[t]{2}{*}{1} & (Constante) & 2.784 & 0.176 & & 15.857 & 0.000 \\
\hline & InPB & 0.319 & 0.046 & 0.564 & 6.990 & 0.000 \\
\hline \multirow{2}{*}{2} & (Constante) & 0.001 & 0.005 & & 0.191 & 0.849 \\
\hline & $\operatorname{var}(\operatorname{InPBt}-1)$ & -0.120 & 0.131 & -0.091 & -0.923 & 0.358 \\
\hline \multirow{2}{*}{3} & (Constante) & 4.097 & 0.084 & & 48.733 & 0.000 \\
\hline & $\ln (\mathrm{CMg})$ & -0.078 & 0.026 & -0.285 & -3.045 & 0.003 \\
\hline \multirow{2}{*}{4} & (Constante) & 0.602 & 0.208 & & 2.893 & 0.005 \\
\hline & $\ln (P L t-1)$ & 0.850 & 0.052 & 0.852 & 16.405 & 0.000 \\
\hline
\end{tabular}

a. Variable dependiente: $\ln (P L t)$

\section{Contraste con el modelo ARIMA}

Estadísticos del modelo

\begin{tabular}{|c|c|c|c|c|c|}
\hline \multirow{2}{*}{ Modelo } & $\begin{array}{c}\text { Número de predic- } \\
\text { tores }\end{array}$ & Estadísticos de ajuste del modelo & \multicolumn{3}{|c|}{ Ljung-Box Q(18) } \\
\cline { 3 - 6 } & $\mathbf{R}$ cuadrado estacionaria & Estadísticos & GL & Sig. \\
\hline $\ln$ (PLt)-Modelo_1 & 1 & 0.728 & 15.931 & 17 & 0.529 \\
\hline
\end{tabular}




\section{Electricity as a Factor of Competitiveness: Empirical Evidence in the Free Customer Market in the Context of Camisea Natural Gas}

Miguel Peralta Neyra ${ }^{1}$

Received: 26/09/2019 Accepted: 18/04/2021 Published: 26/07/2021

\begin{abstract}
The exploitation of Camisea natural gas implied benefits for industries with respect to the electricity rates of which they are large consumers. The national electricity market is based on three prices: busbar price, marginal cost, and free customer price. This study aims to establish whether the advantage of the low price of natural gas is reflected in the price for free customers, who are those who can directly negotiate their electricity tariff with the electricity generators or distributors. This was achieved through a time series analysis with econometric models linking the three national electricity market prices, which indicated the correlation between them. A review of the proposed models, their correlational analysis and the autocorrelation analysis of the residuals revealed a strong correlation between free market price and its first lag, which dissociates a correlation with the other prices; therefore, there is no real transfer of the gas price for a better tariff for the free costumer.
\end{abstract}

Keywords: energetic mix; unregulated customers; busbar price; cost.

\section{INTRODUCTION}

With the enactment of the Ley de Concesiones Eléctricas ${ }^{2}$ (Law No. 25844 , November 19, 1992) the role of the State in the electricity sector changed from one of administration to one of supervision. For this, the roles of existing regulatory entities were reformulated and strengthened, and afterwards others were created with specific tasks. This Law specifies aspects such as the new structure of the sector, existing markets and activities, and price regimes. It also defined the functions and structure of the Comisión de Tarifas Eléctricas ${ }^{3}$ (today known as Gerencia Adjunta de Regulación Tarifaria ${ }^{4}$, which belongs to Osinergmin), the procedures to be followed for concessions, the rights and obligations of the concessionaires, and the functions of the body in charge of the efficient operation of the system (COES). At the same time, the transactions related to price regulation were defined, as well as the procedures for setting rates.

Although the Electrical Concessions Law disintegrated the state monopoly, the subsequent privatization had to make sure that vertical and horizontal integration did not occur; thus, the law was designed with the assignment of this responsibility to the regulatory agency Osinergmin.

On the other hand, some authors see vertical integration as an advantage because it offers the possibility of sharing synergies and risks (Viscusi, Vernon \& Harrington, 2005). Reduced competition, associated with fewer options for consumers and the monopolistic exploitation of a series of specificities such as location, idiosyncratic investments, etc., not only affects the consumer, but also distorts the market. Regarding horizontal concentration, since 2012 the Herfindahl-Hirschman Index $(\mathrm{HHI})$ was less than 1800 , which means that this market was moderately concentrated; it should be mentioned that the $\mathrm{HHI}$ trend is that of reduction (Cuadros \& López, 2016).

\footnotetext{
1 Degree in Mechanical Engineering from Pontificia Universidad Católica del Perú (Lima, Peru). Specialist in natural gas engines and power generation projects. Currently teaching energyrelated courses at Pontificia Universidad Católica del Perú and independent consultant in natural gas projects (Lima, Peru) ORCID: https://orcid.org/0000-0002-7769-7606 Corresponding author: peralta.m@pucp.pe

2 Electricity Concessions Law

3 Electricity Rates Commission

4 Deputy Management of Rate Regulation
} 
The price of electricity for free customers, also called the unregulated price (Morandé \& Soto, 1996), is established through the contracts acquired in the negotiation with the bidders, which in this case are the generators and distributors of the electricity market (Dammert, Mollinelli \& Carbajal, 2011). Most of these free customers are large companies from the mining, smelting, cement, chemical, metallurgical and industrial sectors, among others. The price these customers pay is of great importance, since it is precisely in this customer segment where, according to the regulation, competition has the greatest potential.

Regulated customers, those whose rates are regulated, are under the protection of Osinergmin, which sets the maximum rates for generation, transmission and distribution. Regulated market prices are related to busbar prices, which is the price that generators or distributors guarantee until a physical busbar or supply point located within their network, and marginal costs. Both busbar prices (Osinergmin, 2017) and marginal cost (Resolución de Consejo Directivo Osinergmin No. 013-2016-OS/CD, 2016) are influenced by fuel value, since it is the cause of the differentiation of technology investment and costs; for example, hydroelectric power plants require a large initial investment with a low value of the "fuel" (dam water) for their operation, while diesel turbogenerators require low capital investment with a higher fuel (hydrocarbon) cost against water resources.

Osinergmin has established that the rate for regulated customers must not differ neither more nor less than $10 \%$ of the weighted average of the bid prices in which free customers participate, which means that regulated market prices are sensitive to the free market price (Resolución del Consejo Directivo Osinergmin No. 273-2010-OS/CD, 2010). Thus, it is reasonable to think that if the prices for free customers were manipulated, this would be transferred to the regulated price (De La Cruz \& García, 2002). It must be considered that the electricity suppliers in the free customer market can be, and in fact are, energy suppliers in the regulated market. This study is about the way free market prices are behaving with respect to the regulated market prices (regulated price) and whether Camisea gas really contributes to making the free customer sector more competitive.

It is common to hear that Camisea gas has been considered since the beginning an energy source that improves and will improve the economy of the country; however, there has not been a reliable study of its influence on the improvement of production costs in industries, even though it is known that electricity is a supply with great specific weight in the cost of production.

Since electricity is an important supply in the production process of most industries, especially in mining, smelting, refining, etc., this study aims to establish whether the inclusion of natural gas into the new Peruvian energy mix is really contributing to benefit the industry in general and to make it more competitive through lower rates due to the fact that natural gas is a cheaper fuel than other hydrocarbons. It is re-emphasized that the prices established in the electricity market are free customer prices, regulated prices (which depend on the busbar prices and the marginal cost) and the marginal cost. As the busbar price and the marginal cost are strongly influenced by the fuel cost, there is the need to know whether the benefits of a lower gas price are being passed on to free customers.

The objective of this work is to investigate whether natural gas, as a new source in the energy mix, contributes to improve the competitiveness in the Peruvian industry. For this purpose, significant relationships in the electricity market prices that influence the price of the free customer market will be established. This work also aims to determine at how the lagged free customer prices influence the free customer price, and how the current marginal cost and its lags influence the free customer price.

This work seeks to investigate the relationships that occur within electricity market prices. It seeks to establish whether there is a transfer of the benefit of having a cheaper hydrocarbon, such as natural gas, to an industry that intensively uses electricity in its production process. It will also show how the busbar prices, the marginal cost and its lags, including even the free customer price, influence on the free customer price. This research can be extended to other regulated markets such as telecommunications, clean water service and transportation.

\section{METHODOLOGY}

Since the influence of Camisea gas development in the free client market is an element of analysis in this research, it is necessary to specify when this hydrocarbon began to be used for thermoelectric generation. The Camisea gas project concluded with the start-up of the Malvinas compressor station and the LNG pumping stations in August 2004 (EI 
Comercio, 2014). The generators that at the date of this study use Camisea gas began operating at the end of 2004, and one of the most important is the Etevensa Plant in Ventanilla, which began operations with a simple-cycle application and in the second half of 2006 was consolidated with the entry of its combined-cycle application. Additionally, the large thermogenerators located in Chilca, which are powered exclusively by Camisea gas, started commercial operation as Kallpa I and Chilca I in 2006 and 2007 with their T1 and T2 gas turbines, respectively. All the data presented above allows to have confidence that the sample to be chosen as the study population is the information referring to the electricity market from 2008 onwards, where the operation, dispatch and the market itself is consolidated and stabilized.

This research is based on the analysis of documents provided by Osinergmin and COES, which are the competent authorities that monitor and prepare the statistics related to the performance of the electricity market in Peru.

The information was collected as follows:

Free Market Price. Through the website http:// srvgart07.osinerg.gob.pe/SICLI/principal.aspx, option "Evolución del Mercado Libre", menu "Variable"; then option "Precio medio (ctm/S/./kW.h)"; menu "Clasificado por" and then "Mercado Libre". Lastly, the period to be consulted was seleted.

Busbar Price and Marginal Cost. Through the website http://www.coes.org.pe/Portal/Publicaciones/Estadisticas/, option "Publicaciones", followed by "Estadísticas Anuales"; the folder corresponding to year 2017 was accessed, then "01_EXCEL", "16_COSTOS MARGINALES DE CORTO PLAZO" and finally the file "Gráfico No 16.3_ COSTO MARGINAL PONDERADO Y TARIFA EN BARRA MENSUAL".

This research is quantitative, inductive, basic, longitudinal, correlational and non-experimental. The units of analysis are taken from the reports of the Peruvian electricity market values obtained through the Portal del Sistema de Usuarios Libres de Osinergmin (Osinergmin Free Users System Portal) (http://srvgart07.osinerg.gob.pe/SICLI/principal. aspx) and the COES portal (http://www.coes.org. pe/Portal/Publicaciones/Informes/).

Three correlational models applied to this study and which link the prices in the Peruvian electricity market are presented below:
- Model $1 \quad \ln \mathrm{PL}_{\mathrm{t}}=\alpha+\beta \ln \mathrm{PB}_{\mathrm{t}}+\varepsilon_{\mathrm{t}}$

- Model 2 In PLt $=\alpha+\beta^{*} \ln C M g t+\varepsilon t$

- Model $3 \quad \ln P L_{t}=\alpha+\beta^{*} \operatorname{lnPL} L_{t-1}+\varepsilon_{t}$

Where:

- $\alpha, \beta$ are coefficients.

- $\varepsilon_{\mathrm{t}} \quad$ is defined as a random or stochastic shock.

- In is the natural logarithm function.

- $P L_{t}$ is the free market price in period $t$.

- $\mathrm{PL}_{\mathrm{t}-\mathrm{n}}$ is the free market price with lag $\mathrm{n}$.

- $P B_{t}$ is the busbar price in period $t$.

- $\mathrm{CMg}_{t}$ is the natural logarithm of the marginal cost in period $t$.

If the variability of the data grows with the level of the data, that is, if there are changes in the data that causes that the time series fluctuates vertically from one period to another, (for example, if the minimum and maximum of the series increases or decreases from one year to the next one), it will be necessary to make a correction, since it would represent a problem; this is usually done through a logarithmic transformation, applying natural logarithms to the observations and achieving stationarity in the variance (Chong \& Aguilar, 2016).

The study population is the Peruvian electricity market, and for the sample size, the prices and costs of the electricity market from February 2008 to December 2016 have been selected. These values are shown in Table 1.

\section{RESULTS}

For the correlational analysis, software IBM SPSS Statistics 23 was used and Pearson and $R^{2}$ correlation tests plus Durbin Watson's correlation test were considered (Daza, 2016). The results of each of the proposed models are shown in Appendix A.

Table 2 shows the values of the coefficients, constants and statistics that were considered for the present evaluation.

The Pearson correlation coefficient explains the linear dependence between two quantitative random variables: when its values are closer to 1 , a strong relationship between the variables is evident. Of the three models studied, it was observed that the one with the highest correlation with the variables considered for its own case is model three, since it was the one that obtained the highest Pearson correlation coefficient; this result was confirmed 
Table 1. Free market prices, Busbar Price and Marginal Cost (ctv \$/kW.h) of period February 2008 to December 2016.

\begin{tabular}{|c|c|c|c|c|c|c|c|}
\hline Month/year & Free Market & $\begin{array}{c}\text { Regulated } \\
\text { Busbar Price } \\
\text { Lima }\end{array}$ & Marginal Cost & Month/year & Free Market & $\begin{array}{c}\text { Regulated } \\
\text { Busbar Price } \\
\text { Lima }\end{array}$ & Marginal Cost \\
\hline Feb-08 & 49.4999 & 43.5792 & 18.3336 & Aug-12 & 58.5272 & 50.8816 & 35.0912 \\
\hline Mar-08 & 48.2512 & 45.0487 & 20.8422 & Sep-12 & 58.9011 & 52.1772 & 36.4090 \\
\hline Apr-08 & 50.2247 & 44.4016 & 20.9027 & Oct-12 & 59.5905 & 52.4412 & 28.7611 \\
\hline May-08 & 53.0576 & 44.1790 & 47.8629 & Nov-12 & 58.0696 & 51.3352 & 14.3501 \\
\hline Jun-08 & 61.7507 & 44.8090 & 148.8524 & Dec-12 & 57.7426 & 51.7424 & 13.7504 \\
\hline Jul-08 & 57.8397 & 40.1050 & 235.3823 & Jan-13 & 59.1261 & 52.2300 & 19.3484 \\
\hline Aug-08 & 68.9169 & 41.5437 & 157.8758 & Feb-13 & 60.2324 & 52.7082 & 31.4054 \\
\hline Sep-08 & 68.0654 & 39.8481 & 185.2081 & Mar-13 & 57.7833 & 51.3073 & 19.7176 \\
\hline Oct-08 & 54.5291 & 40.9945 & 63.3530 & Abr-13 & 59.7752 & 51.8077 & 18.7835 \\
\hline Nov-08 & 54.5909 & 41.1696 & 60.6917 & May-13 & 59.3034 & 50.2321 & 27.1427 \\
\hline Dec-08 & 55.0423 & 39.1783 & 81.7817 & Jun-13 & 58.1775 & 50.5006 & 26.6098 \\
\hline Jan-09 & 48.1669 & 37.7910 & 28.8897 & Jul-13 & 58.7349 & 50.2361 & 44.8607 \\
\hline Feb-09 & 49.6494 & 37.5074 & 42.3859 & Aug-13 & 58.5656 & 49.8932 & 34.7275 \\
\hline Mar-09 & 47.3418 & 37.8923 & 26.4559 & Sep-13 & 58.6253 & 48.2247 & 28.2676 \\
\hline Apr-09 & 45.7491 & 41.1450 & 25.4261 & Oct-13 & 58.0340 & 50.4502 & 19.4460 \\
\hline May-09 & 46.6875 & 39.7411 & 28.6713 & Nov-13 & 58.7762 & 50.2010 & 23.0011 \\
\hline Jun-09 & 49.5528 & 39.9566 & 65.7041 & Dec-13 & 58.6221 & 50.4372 & 24.8975 \\
\hline Jul-09 & 48.1972 & 39.8324 & 41.2193 & Jan-14 & 59.3035 & 50.1908 & 21.3909 \\
\hline Aug-09 & 47.2772 & 39.4824 & 33.8807 & Feb-14 & 60.1234 & 55.0746 & 29.8533 \\
\hline Sep-09 & 47.7056 & 40.1442 & 36.2248 & Mar-14 & 59.6457 & 54.4080 & 34.3143 \\
\hline Oct-09 & 46.8315 & 40.5292 & 19.7863 & Apr-14 & 60.7635 & 55.0021 & 28.1039 \\
\hline Nov-09 & 49.6828 & 40.7031 & 20.3717 & May-14 & 59.7786 & 54.8269 & 25.4199 \\
\hline Dec-09 & 53.2740 & 38.5393 & 17.2423 & Jun-14 & 60.6186 & 54.8215 & 30.9638 \\
\hline Jan-10 & 53.2165 & 39.0021 & 23.1519 & Jul-14 & 60.1494 & 54.6584 & 24.9118 \\
\hline Feb-10 & 53.7494 & 39.8040 & 24.5490 & Aug-14 & 61.0708 & 54.2496 & 27.4186 \\
\hline Mar-10 & 50.7876 & 39.1649 & 21.9672 & Sep-14 & 61.2980 & 53.7230 & 23.8568 \\
\hline Apr-10 & 53.8770 & 39.8972 & 16.6041 & Oct-14 & 60.9968 & 52.4992 & 17.9716 \\
\hline May-10 & 47.0575 & 37.0416 & 18.1607 & Nov-14 & 60.2288 & 50.0767 & 23.4544 \\
\hline Jun-10 & 47.9560 & 36.9624 & 20.4322 & Dec-14 & 59.7798 & 49.3496 & 15.1614 \\
\hline Jul-10 & 48.3525 & 37.1588 & 19.8814 & Jan-15 & 59.9644 & 48.8229 & 14.1122 \\
\hline Aug-10 & 48.8606 & 37.5823 & 22.8932 & Feb-15 & 60.9203 & 50.9184 & 16.2237 \\
\hline Sep-10 & 50.3119 & 38.0923 & 23.8426 & Mar-15 & 58.9541 & 49.6404 & 17.0768 \\
\hline Oct-10 & 49.3977 & 37.6483 & 24.2287 & Apr-15 & 59.5711 & 49.6373 & 13.1058 \\
\hline Nov-10 & 50.0032 & 37.6004 & 23.1013 & May-15 & 58.8955 & 55.9126 & 14.8277 \\
\hline Dec-10 & 50.0807 & 37.2586 & 18.7580 & Jun-15 & 59.0806 & 55.9179 & 16.9142 \\
\hline Jan-11 & 51.6746 & 37.7511 & 17.4892 & Jul-15 & 58.2973 & 58.0145 & 10.9362 \\
\hline Feb-11 & 54.5046 & 38.4420 & 21.7420 & Aug-15 & 57.9421 & 57.1395 & 21.4953 \\
\hline Mar-11 & 53.4987 & 37.6326 & 21.6263 & Sep-15 & 59.0000 & 58.0990 & 14.4870 \\
\hline Apr-11 & 55.0812 & 39.2423 & 17.9153 & Oct-15 & 58.0086 & 57.3931 & 14.2471 \\
\hline May-11 & 53.2369 & 41.8831 & 18.7874 & Nov-15 & 57.5160 & 55.5090 & 11.5861 \\
\hline Jun-11 & 53.2595 & 42.2603 & 25.8559 & Dec-15 & 56.0025 & 54.0816 & 11.3972 \\
\hline Jul-11 & 53.8045 & 42.4235 & 20.4480 & Jan-16 & 54.7017 & 56.6792 & 10.9870 \\
\hline Ago-11 & 54.0971 & 42.4162 & 31.5137 & Feb-16 & 53.6933 & 54.9479 & 12.4248 \\
\hline Sep-11 & 55.4634 & 42.7455 & 33.6255 & Mar-16 & 50.8376 & 55.8568 & 12.3632 \\
\hline Oct-11 & 53.5310 & 42.4366 & 27.0617 & Apr-16 & 52.0433 & 54.5576 & 13.2597 \\
\hline Nov-11 & 54.9701 & 43.2516 & 28.5765 & May-16 & No data & No data & 19.9034 \\
\hline Dec-11 & 53.7427 & 43.1351 & 21.5721 & Jun-16 & No data & No data & 60.3911 \\
\hline Jan-12 & 55.3760 & 43.4912 & 20.9235 & Jul-16 & 53.7055 & 55.1906 & 34.1345 \\
\hline Feb-12 & 56.1628 & 44.3116 & 23.7337 & Aug-16 & 53.3021 & 56.7516 & 18.9263 \\
\hline Mar-12 & 56.6856 & 43.6561 & 39.8348 & Sep-16 & 52.8905 & 56.3791 & 27.5588 \\
\hline Apr-12 & 56.1615 & 44.1915 & 26.6761 & Oct-16 & 51.5354 & 56.4675 & 17.9264 \\
\hline May-12 & 58.3578 & 49.7053 & 27.1751 & Nov-16 & 51.8879 & 58.8807 & 27.5979 \\
\hline Jun-12 & 59.0050 & 50.1692 & 45.5229 & Dec-16 & 50.9509 & 58.8439 & 23.0784 \\
\hline Jul-12 & 58.6341 & 51.0439 & 58.0489 & & & & \\
\hline
\end{tabular}

Source: Prepared by the author based on the information of http://srvgart07.osinerg.gob.pe/SICLI/principal.aspx and http://www.coes. org.pe/Portal/Publicaciones/Estadisticas/ 
by the coefficient of determination $R^{2}$. This model, confronted with the real values, produces residuals that must also be analyzed to rule out an autocorrelation, thus, if autocorrelation is found among the residuals, there is certainty that the model is not adequate to explain the relationships of the variables and it is presumed that a variable was not taken into account and therefore it must be included. The Durbin Watson is the statistic that shows with certainty if the residuals of the samples are not correlated and, for model 3 , this value is in the no autocorrelation area so the model presented produces no autocorrelated residuals and better explains the relationship of the variables under study. The ARIMA model was run as a contrast test to validate the model.

Table 2. Results of the Proposed Models in Software IBM SPSS Statistics 23.

\begin{tabular}{|l|c|c|c|}
\hline \multicolumn{1}{|c|}{ Values } & \multicolumn{3}{c|}{ Models } \\
\hline & 1 & 2 & 3 \\
\hline$\alpha$ & 2.784 & 4.097 & 0.602 \\
\hline$\beta$ & 0.319 & -0.078 & 0.850 \\
\hline$R^{2}$ & 0.564 & 0.081 & 0.725 \\
\hline Adjusted $R^{2}$ & 0.318 & 0.072 & 0.722 \\
\hline Pearson & 0.311 & -0.285 & 0.852 \\
\hline$F$ & 48.863 & 9.270 & 269.120 \\
\hline Durbin Watson & 0.387 & 0.093 & 2.133 \\
\hline
\end{tabular}

Source: Prepared by the author based on the results of software SPSS.

\section{DISCUSSION}

The model proposed must also demonstrate that there is no autocorrelation; for that, the Durbin Watson statistic (DB) provided by SPSS software was used (Gujarati, 1997). According to Table 2, the DB of the model presented is 2.132 and must be compared with the null hypothesis, which indicates that there is no autocorrelation for the values of DB between $d_{u}$ and $4-d_{u}$ which, for $5 \%$ significance, gives a value of $d_{u}$ equal to 1.694 and a value of $4-d_{u}$ equal to 2.346 . Therefore, the null hypothesis of no autocorrelation is validated. The ARIMA model provides a value of the Ljung Box statistic of 0.529 , higher than the minimum accepted value of 0.05 , so the model is valid.

\section{CONCLUSIONS}

There is a decoupling of the free customer price with busbar prices and marginal costs, since although these values are dependent of fuel cost, the free customer price is not related to the price of natural gas.
The model that best explains the relationship of the free customer price with electricity market prices is precisely model 3 , which links the one-period lagged free customer price; in the study, that period is one month.

Large industries should have the same benefits as the generators that, for example, are able to generate their own energy. Natural gas rates for industrial consumption and for generation are differentiated and, likewise, the largest consumers of gas for generation have better rates. The deconcentration of high voltage lines, thanks to self-generation, can improve the quality of the service, which is quite poor in extreme areas of the lines and, in turn, can be reinforced with the injection of surpluses from industries.

\section{REFERENCES}

[1] Chong, M., \& Aguilar, R. (2016). Proyección de series de tiempo para el consumo de la energía eléctrica a clientes residenciales en Ecuador. Revista Tecnológica ESPOL, 29(1), 56-76.

[2] El Comercio (April 30, 2014). El recorrido de Camisea, un proyecto que esperó dos décadas. El Comercio.

[3] Cuadros, A., \& López, G. (2015). Análisis de condiciones de competencia en el mercado de generación eléctrica en el Perú, periodo 2010 2014. Retrieved from https://www.coes.org.pe/ Portal/Publicaciones/Articulos/

[4] Dammert, A., Mollinelli, F., \& Carbajal, M. A. (2011). Fundamentos Técnicos y Económicos del Sector Eléctrico Peruano. Lima, Peru: Osinergmin.

[5] Daza, J. (2016). Análisis Estadístico SPSS Statistics. Lima, Peru: Megabyte SAC.

[6] De La Cruz, R. \& García, R. (February, 2002). Mecanismos de Competencia en Generación de Energía y su impacto en la Eficiencia: El caso peruano.. Retrieved from https://cies. org.pe/sites/default/files/investigaciones/ mecanismos-de-competencia-en-generacionde-energia-y-su-impacto-en-la-eficiencia.pdf

[7] Gujarati, D. (1997). Econometría. Sante Fé de Bogotá, Colombia: McGraw-Hill.

[8] Ley No. 25844 (November 19,1992). Ley de Concesiones Eléctricas. Diario Oficial El Peruano. Retrieved from https://www. osinergmin.gob.pe/cartas/documentos/ electricidad/normativa/LEY_CONCESIONES_ ELECTRICAS.pdf 
[9] Morandé, F., \& Soto, R. (1996). El Mercado de clientes no regulados en la industria eléctrica. ILADES/Georgetow .

[10] Osinergmin (March 16, 2017). Procedimiento de Fijación de Precios en Barra Periodo mayo 2017 - abril 2018.

[11] Resolución del Consejo Directivo Organismo Supervisor de la Inversión en Energía y Minería Osinergmin No. 273-2010-OS/CD. Aprueban "Procedimiento para la comparación de precios regulados". Diario Oficial El Peruano (2010). Retrieved from http://www.osinergmin.gob. pe/newweb/uploads/Publico/Resoluciones/ ConsejoDirectivo/2010/OSINERGMIN\%20 No.273-2010-OS-CD.pdf
[12] Resolución de Consejo Directivo Organismo Supervisor de la Inversión en Energía y Minería Osinergmin No. 013-2016-OS/CD. Diario Oficial El Peruano. Retrieved from http://www. osinergmin.gob.pe/Resoluciones/pdf/2016/ OSINERGMIN\%20No.013-2016-OS-CD.pdf

[13] Viscusi, W., Harrington, Jr., J., \& Vernon, J. (2005). Economics of Regulation and Antitrust. Cambridge, MA, United States: The MIT Press. 


\section{APPENDIX}

Appendix A. Results of software IBM SPSS Statistics 2 for the proposed models.

Summary of the Models ${ }^{(e)}$

\begin{tabular}{|c|c|c|c|c|c|}
\hline Model & $\mathbf{R}$ & R-Squared & $\begin{array}{c}\text { Adjusted R- } \\
\text { Squared }\end{array}$ & Pearson & Durbin-Watson \\
\hline 1 & $0.564^{(\mathrm{a})}$ & 0.318 & 0.311 & 0.564 & 0.387 \\
\hline 2 & $0.091^{(\mathrm{b})}$ & 0.008 & -0.001 & -0.091 & 2,232 \\
\hline 3 & $0.285^{\odot}$ & 0.081 & 0.072 & -0.285 & 0.093 \\
\hline 4 & $0.852^{(\mathrm{d})}$ & 0.725 & 0.722 & 0.852 & 2,133 \\
\hline
\end{tabular}
a. Predictors: (Constant), InPB
b. Predictors: (Constant), var(InPBt-1)
c. Predictors: (Constant), $\ln (\mathrm{CMg})$
d. Predictors: (Constant), In(PLt-1)

\section{Coefficients $^{(\mathrm{a})}$}

\begin{tabular}{|c|c|c|c|c|c|c|}
\hline & \multirow[t]{2}{*}{ Model } & \multicolumn{2}{|c|}{ Unstandardized coefficients } & \multirow{2}{*}{$\begin{array}{c}\begin{array}{c}\text { Standardized } \\
\text { Coefficients }\end{array} \\
\text { Beta } \\
\end{array}$} & \multirow[t]{2}{*}{$\mathbf{T}$} & \multirow[t]{2}{*}{ Sig. } \\
\hline & & B & Standar Error & & & \\
\hline \multirow[t]{2}{*}{1} & (Constant) & 2.784 & 0.176 & & 15.857 & 0.000 \\
\hline & $\ln \mathrm{PB}$ & 0.319 & 0.046 & 0.564 & 6.990 & 0.000 \\
\hline \multirow{2}{*}{2} & (Constant) & 0.001 & 0.005 & & 0.191 & 0.849 \\
\hline & $\operatorname{var}(\operatorname{lnPBt}-1)$ & 0.120 & 0.131 & -0.091 & -0.923 & 0.358 \\
\hline \multirow{2}{*}{3} & (Constant) & 4.097 & 0.084 & & 48.733 & 0.000 \\
\hline & $\ln (\mathrm{CMg})$ & -0.078 & 0.026 & -0.285 & -3.045 & 0.003 \\
\hline \multirow{2}{*}{4} & (Constant) & 0.602 & 0.208 & & 2.893 & 0.005 \\
\hline & $\ln (P L t-1)$ & 0.850 & 0.052 & 0.852 & 16.405 & 0.000 \\
\hline
\end{tabular}

a. Dependent variable: $\ln (P L t)$

\section{Contrast with ARIMA model}

\section{Model Statistics}

\begin{tabular}{|c|c|c|c|c|c|}
\hline \multirow{2}{*}{ Model } & \multirow{2}{*}{ Number of Predictors } & Adjustment Statistics of the Model & \multicolumn{3}{|c|}{ Ljung-Box Q (18) } \\
\cline { 3 - 6 } & & Stationary R square & Statistics & GL & Sig. \\
\hline In(PLt)-Modelo_1 & 1 & 0.728 & 15.931 & 17 & 0.529 \\
\hline
\end{tabular}

